\title{
6. COORDINATES OF THE INTERPRETATION
}

\section{Luminiţa Ciobanu $^{15}$}

\begin{abstract}
In the process of study, a number of native but also acquired skills are involved which helps to progress and access to a superior artistic level. Internal hearing, sensitivity, motivation, interest, purpose and charisma are part of the native but educative capacities of the individual, as well as attention, operative thinking and memory capacity.
\end{abstract}

Key words: instrumental act, internal hearing, sensitivity, motivation, interest

\section{Introduction}

The instrumental act is associated with a psychic process whose elements are internal hearing, thought, memory, attention, motivation, will, affectivity and instrumental skills.

Internal hearing is a sum of elements necessary for knowledge such as sensations and auditory perceptions, but also the auditory imagination on the basis of which auditory representations are formed. The receiver processes the elements of the sound - the frequency, the amplitude and the shape - to be then reflected and conscious in the form of psychological correlations, which leads to the formation of different sides of the hearing - intensity hearing, height, timbre - sides that can grow unequally, even for the same individual. In the intensity hearing we distinguish different degrees of development. Thus, in the case of absolute sensitivity, the subject is able to respond to the minimum intensity of a sound emitted in natural conditions.

Differential sensitivity is the ability of the receiver to sense a minimum difference between two intensities. This translates into the absolute threshold of the sensation, the minimum amplitude of a sound, capable of triggering the auditory analyzer response. Differential threshold is the minimum intensity difference between two sounds, capable of generating a sensation.

The Weber-Fechner Law, which says that the physiological intensity of the sound (perceived by the ear) increases with the logarithm of its physical intensity, and the sounds are perceived at a lower intensity than the one with which they are emitted. This translates into the interpretation process by the need for increased contrasts so that they can be clearly perceived by the listener's hearing aid.

Lecturer PhD., „George Enescu” National University of Arts from Iaşi, Romania, email: luminita.ciobanu@gmail.com 


\section{Discussions}

Consideration will also be given to the lasting capacity, different between the serious and the acute sounds, due to the harmonic content and the emission wavelength. In the formation of future musicians, the creation of the stamps ideal is of particular importance, a process in which the process of comparison between the sounds of different instruments or voices is really useful. The basis of internal hearing is based on hearing. This translates into reflecting intuitive images of previously perceived sound material and the upper level of internal hearing means that the auditory imagination can build original sound patterns based on the creative processing of representations.

In the process of forming the musician a special role belongs to the motivation. The degree of motivation is adjusted according to the nature and importance of the goal, and it is important to avoid the extremes of motivation so that it does not hinder the effectiveness of the action. Thus, in the face of a low-complex task, motivation, especially among young people, tends to fall backwards, generating degrees of underestimation or over-evaluation of the action. The law of Yerkes and Dodson thus sets out this phenomenon - the ratio between increasing the difficulty of the task and the variation in the intensity of the motivation must be inversely proportional to achieve optimal performance ${ }^{16}$.



Arousal

Every human action, the more a performance, involves some motivations and interests. The reason is the awareness of needs, needs and is expressed through an image or idea that fulfills the role of impulse to a particular action. Reasons can be of several types

- Extrinsical - being characterized by elements related to environmental or social factors

\footnotetext{
${ }^{16} \mathrm{https} / / / \mathrm{www}$.google.ro/imgres?imgurl=https://upload.wikimedia.org/wikipedia/commons/thumb/1/1e/Hebbian YerkesDodson.svg/2000px-
} 
- Intrinsic - they result in the internalization of the outer motifs

- Stimulators - these include positive external actions such as encouragement, praise

- Inhibitors - are negative actions - indifference, fear, punishment.

Interest is a general, stable and broad meaning, with latent attention, while attention is an interest in action. Interest cannot be created by simple action, such as going through a musical material, but it requires a series of specific and motivational skills to deepen that action. In this respect, the teacher has to find and use different means and adapt to the individual personality to develop motivation - eg. auditions, recitals, appreciations, discussions on some aspects of art. The goal is the object of anticipated action and being subordinate to motivation. The results, positive or negative, will directly influence the purpose. If the aspiration level is on the rise, the goal will become clearer, more stable, and vice versa. Another phase of the voluntary act is the decision. Its stability is closely related to the oscillations during preparation. A strong will makes possible to prioritize goals - small ones will subordinate those with profound significance.

The final phase of the voluntary act is the enforcement of the judgment, which implies its maximum concentration, with maximum efficiency. The startup phase of the study does not provide a materialization of the need for this action, so that a strong will is needed to compensate for the lack of need. Such a constraint is required, especially during the preparation of an exam or recital. A particularly important aspect governing the act of interpretation is affectivity. A factor of this is emotionality - the one that represents the dynamic vector of personality and helps him in the intense work of the instrument, the effortless mobilization of the necessary energy and the persistence in the study activity. A degree of normal emotions will reflect the performance of the performer on stage and generally under stress. Hyper-emotions leads to the inability to solve situations considered normal for the average of individuals, resulting in results generally inferior to the possibilities of that person. The timid has a normal working capacity, but it manifests itself with difficulty in the inter-human relations, especially with those that are of value to him.

\section{Results}

In the act of interpretation, attention is also very important, in particular stability, maintaining it for a long time and throughout a pursued goal. Stability of attention differs from individual to individual, being conditioned by the constitution of the central nervous system, but also by an educated and selfeducating quality. Thinking operatively is the first step towards the creative thinking that an artist needs so much. By contrast, its absence reveals an inert and rigid person. Memorization, voluntary or involuntary (according to the intent criterion), is the process of imprinting the information in the musical text so that it can then be played in creative mode. In the process of memorization, 
the individual calls for 3 logical operations - analysis, synthesis and comparison. Other factors such as motivation, affection, and technical abilities of the individual are associated with this process. Hermann Ebbinghaus ${ }^{17}$ argues that forgetfulness is massive at the beginning of the memory process, but in the course of time it is becoming slower.

It therefore follows that memorization must be done on a voluntary, logical and precise basis, and the technique of link consolidation must be very rigorous. Through numerous repetitions, the skills turn into automated parts of the instrumental act. These are of an intellectual, motor and sensorial type, and for automation they will go through 3 psychological phases: the irradiation phase (where the faults are numerous and the energy consumption is maximum, the inhibition phase - when the excitation in the cerebral cortex decreases and the flowing course with the reduction of unnecessary movements and the stereotype phase, in which the movement is achieved with minimal effort and maximum efficiency.

\section{Conclusions}

The study model, which must be clear and precise, must have a great importance in the formation of skills, and repetitions must be carried out in varying parameters - time (rarely, quickly), quantity (on fragments, global), quality (expressive - arid) etc. This type of work favors analysis and synthesis as well as systematization capacity. through repetition and practice lead to the formation of skills and knowledge and then to the skill of $\mathrm{p}$ superior, which involves motor intelligence, creativity and the ability to solve complex problems.

\section{References}

1. Ciocan, D. (2006) A semiotic theory of musical interpretation, Editura UNMB, Bucuresti

2. Pașca, E. M. (2008) Strategies of disciplinary and interdisciplinary approach to musical education in simultaneously primary education Editura Artes, Iasi

3. Răducanu, M. D. (1994) Principles of instrumental didactics, Editura Moldova, Chisinau

4. https://www.verywellmind.com/what-is-the-yerkes-dodson-law-2796027

5. https://en.wikipedia.org/wiki/Hermann_Ebbinghaus

${ }^{17}$ https://en.wikipedia.org/wiki/Hermann_Ebbinghaus 